\title{
LV-PP-2-2
}

\section{The correlation between preoperative volumetry and actual graft weight of liver according to the donor age}

Eun Jin KIM, Hyun Jeong KIM, Jae Geun LEE, Dae-Hoon HAN, Gi Hong CHOI, Jin Sub CHOI, Myoung Soo KIM, Soon II KIM, Dong Jin JOO*

Department of Surgery, Yonsei University College of Medicine, Seoul, Korea

Introduction: Liver volume assessment is essential to provide adequate graft volume (GV) to recipients and ensure safety to donors in living donor liver transplantation. This study aims to compare the GV calculated by preoperative liver volumetry and graft weight (GW) measured during surgery according to the donor age and the influence of GRWR mismatch on patient survival.

Methods: Data from 771 living donors between 2005 to 2020 in Severance hospital were reviewed. We defined GW mismatch as a discrepancy $\geq 10 \%$ between preoperatively estimated and actually measured GRWR. Donor groups were divided into 6 groups based on ten year age interval and data were compared according to their age. Recipient survival outcomes were analyzed in accordance with the GRWR mismatch.

Results: The proportion of GRWR mismatch donors was not different across the donor age groups ( $47 \%$ vs. $43 \%$ vs. $48 \%$ vs. $45 \%$ vs. $39 \%$ vs. $60 \% ; p=0.778)$. However, actual GRWR was significantly smaller than estimated GRWR as donor was younger $(p=0.002)$. Total $29(3.7 \%)$ recipients received liver whose GRWR $<0.8$ and $24 \%$ of them $(n=7)$ underwent portal flow modulation (splenic artery ligation, splenectomy, or renal vein ligation) during transplantation surgery. Overall survival did not differ between GRWR $<0.8$ and $\geq 0.8$ groups.

Conclusions: Actual liver GW tends to smaller than estimated GV as donor is younger. GRWR mismatch was not affect overall survival of recipients. 